\title{
Ticket Collectionwith Destination Prediction in Bus Services in Urban Areas using Time Based Predictive Algorithm
}

\author{
S.Jayakumar, M.Srivathsan, S.Samundar Ahmed, Dhanish Kumar S M, M.Karthikeyan
}

\begin{abstract}
In the new age of automation and machine assisted function of the human way of life people still tend to notice verification and checking of tickets in local land transport such as trains and buses to still be operated by man. This project is a proposal of a new platform and method to book these tickets of buses on a local level. This can lead to decrease in the overcrowding of buses, easy time management of commuters, and smooth functioning of the bus business. Initially the bank details of the passenger must be linked to the app.Machine learning predictive parsing algorithm in combination with data mining features enable the prediction of the passengers to and fro details on a daily and timely basis. Then a SMS alert for ticket payment proof is sent to the user. In admin side, they calculate amount details using this application. Per day amount details of specific route or bus can be calculated by accessing the database. There is also a provision where the IMEI numbers of the consumers is collected. Through GPS system the IMEI numbers of the mobiles inside the bus is checked with the IMEI numbers of those in the database. Ticket defaulters are identified if the IMEI numbers are not present in the database. The entire trail of the transit is on a non-paper sever.
\end{abstract}

Keywords-GPS,IMEI,Ticket.

\section{INTRODUCTION}

The concept of having a paper ticket is slowly being replaced in all forms of lifestyle. All the way from train ticket to tickets to a movie or concert, all of them are being replaced by and E-ticket. Then we stopped to think. Why stop at all those, maybe this concept needs changing on a day to day basis. Thus we come up with this project to make it happen even in the daily commute of metropolitan busses. Route planning, traveler information and functional updates are becomingimportant constituents of public transport systems. It helps people navigate preset ecosystems by givinga doorway to service disruptions along within taxations regarding time delays. But, one feature that systems lack is a way of scrutinizing the information they offer with reason to provide personalized and real-time notifications to each traveler.

Revised Manuscript Received on October 12, 2019

S.Jayakumar, Assistant Professor, Department of Computer Science And Engineering ,SRM Institute of Science And Technology.

M.Srivathsan, Student, Department of Computer Science And Engineering,SRM Institute of Science And Technology.

S.Samundar Ahmed, Student, Department of Computer Science And Engineering, SRM Institute of Science And Technology.

Dhanish Kumar S M,Student, Department of Computer Science And Engineering, SRM Institute of Science And Technology.

M.Karthikeyan ,Student, Department of Computer Science And Engineering, SRM Institute of Science And Technology.
Mining thehistory of journeys of various users, collected by preprogrammed ticketing units, unlocks the possibilities to address this crater. In this work, we analyze one such dataset of travel history. In broad way of functioning, every bus is monitored by a certain ticket checker. The conductor collects money from each traveler and be responsible forissuing tickets. Initially tokens and printed papers. Nowadays, printers are used for printing tickets. This process has many drawbacks. The traveler must keep the ticket throughout the journey. The time taken for ticketing is comparatively more.Also large quantities of paper are required for printing the ticket. Thesedays, ticket collectors aretrained in operatingticket issuing machine. For example, if atraveler wishes to commute the bus, he willhave to have paper currency with him. Then the checker will receive the money and he has to distribute tickets to all passengers. This require lot of human resource and is a waste of time and energy. Handheld ticketing machine is also slow and needs to train a person extensively to operate it.

In this project, we are coming up with ideas to use QR reader for bus ticket. Users can scan $\mathrm{QR}$ reader instead of ticket. In this app, after registration process if the profile, we have to attach our bank details through in this app. Then whenever the passenger is boarding the bus, they have a choice for from and to location. Then it will generate amount details for per head. After that we have to obtain passenger details. Passenger's details mean count. Then we can scan QR code. So directly money will transfer from our bank details. Then we can get SMS alert for ticket payment proof. Then admin side will calculate amount details through using web application. Then they can calculate per day amount details for bus ticket information. Then admin can generate per day 2.5 hours report for checking that crowd condition.

The current system is almost completely handled by physical man power. From collecting currency for the fare, to counting and extracting the total and also accounting for the tickets sold, is done by hand. In the proposed system all tickets are paper-less which makes the trail of all those tickets and finances also paper-less. A set of 100 tickets per route and price point is called a block. Now the blocks have to be accounted for. The number of blocks allocated are also limited. So in this system these accounts are easily registered by accessing the database for the booked records. The app along with the web based system helps to reduce all this paper trail to almost negligible.

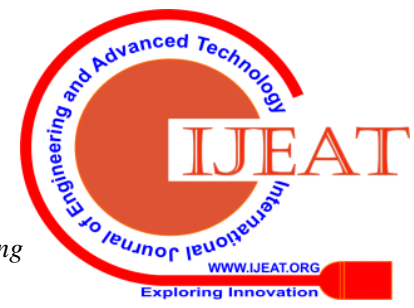




\section{OBJECTIVE}

The main theme of this particular project is to help an individual book ticket easily for local travel. An automatic suggestion of the destination makes the process of booking tickets easier. This system also defines the number of persons per bus. By getting the information about requirement the number of busses to be allocated for a particular time and route can be managed more efficiently. The current system does not have an effective way for identifying the ticket defaulters. This system automatically detects the ticket defaulters. The main motive is to help the people. The main motive is to completely eradicate the use of paper. The current system uses distribution of ticket which is made up of paper. Loads of paper and ink can be reduced by this method. There is no need to worry about the change for tickets in this system. The exact amount of theticket gets automatically debited from the user's bank account. Thus by using these methods we can provide users a repetitive type of commuting where their only job is to choose their destination of travel and then end up boarding the bus. Everything else that they might have needed to do in the past will be eliminated

\section{LITERATURE SURVEY}

The most apt reference comes from "RFID and Android Based Smart Ticketing and Destination Announcement System by Dr. PrasunChowdhury, PoulamiBala, DiptadeepAddy, SumitGiri, Aritra Ray Chaudhuri from Department of $\mathrm{ECE}^{[2]}$ states a lot on the current system of automation. Although this is implemented in a lot of countries it is still not very feasible for a country as vast and populous as India. It shows how the use of RFID can help benefit and automatize the travel on local buses. Travelers have to use the system of RFID and authenticate themselves before entering the bus. Although this is automatic it still requires a physical present member of the bus. It requires a whole team to find defaulters. Adding to all this, something which has much to offer is the Android application, developed as a user interface. Their paper suggests building an RFID system victimization ARM processor which will establish passengers publically transport additionally as will all accounting purpose associated with movement expenses. We, on the other hand suggest that we use a more modern solution to this modern problem. We propose the person using the service will in all possibility, possess a phone. This phone can serve as an entire identity in itself. Even without the QR, the GPS and IMEI will help out in identification and ticketing. So this GPS attribute of our project is discussed in "GPS Supported City Bus Tracking \& Smart Ticketing System" by Ajay Shingare, AnkitaPendole, Nikita Chaudhari, Parikshit Deshpande and Prof. SamadhanSonavane Dept. Of Computer Engineering. ${ }^{[3]}$ This paper emphasizes the importance and easy availability of GPS. This system could widely be used in tracking and also safety and authenticity of the system and its users. Their positive identification primary ticketing module swaps the paper to the sensible hand-held machine for group action. The micro-scale smart ticketing device will be enabled with dynamic roust in accordance with the terminal. The device has an added advantagewith GPS technology and usage of GSM has created offerings with needed necessary tweaks that make it terribly efficient. This system is also implemented in some regions. Our counter proposal to this already efficient system is to be able to use the device IMEI instead of the selective GSM service. We intend to use the GPS embedded with IMEI. Any phone can be tracked using it's IMEI. We plan on using IMEI readers in busses. Thus, if a GPS id does not match with IMEI defaulters can be routed. Now comes the grave problem of ticket allocation. For this the golden rule of first come first serve is used. Now to be able to do this we use direct reference to A First-ComeFirst-Serve Bus-Allocation Scheme Using Ticket Assignments by D. K. SHARMA and S. R. AHUJA. This is the only paper dated almost 30 years ago, but we still believe that some systems deserve a slight touch of technology long forgotten in a more efficient way. The classic first come first serve will also come equipped with a waiting list system and will also have standing spaces according to the class of the bus available at that time. This would also help the admin side to study and frequent busses according to time of demand and rest some busses at times of low demand. Next comes the idea to use a mobile app and its implementation. Mobile Enabled Bus Tracking and Ticketing System by Suresh Sankarananrayanan and Paul Hamilton from Computing and Information systems and Delta Supply Co Ltd Institute Technology of Brunei Kingston and Brunei Darussalam Jamaica ${ }^{[4]}$, proposed to replace the existing RFID with mobile apps. The difference between here and there is mainly the population of users. So just a mobile app wouldn't be feasible in our scenario. Thus the app we build is more complex and includes much more attributes and functions that this proposal. Our app must serve as an entire identity such that even if the person is not present and the mobile device is it is still acting as the person itself. Now the idea of e ticketing of bus tickets in city to city travel is a fairly common idea. What SanamKazi Assistant Professor, Dept. of IT MHSSCOE,Mumbai, MurtuzaBagasrawala Student (B.E), FarheenShaikh Student (B.E) and AnamtaSayyed Student(B.E) Dept. of IT ,Mumbai suggest in their paper "Smart E-Ticketing System for Public Transport Bus" is that, in the era of Digital India (a campaign launched bythe Government of India) and Cashless Economy[1], cheaper form of transport has to blend into the technology advancement. Global Positioning System is being utilized for following of bus location and route planning. This has been target accomplished in some towns in India(ex: Ahmadabad), where the government is responsible for developing a GPS tracking enabled public bus system to fulfill the transportation wants and increasing needs for the protection and reliability of the system. BRTS wants to accomplish a mixof the capability and velocity of an underground along with the flexibleness, lower value and ease of a bus system. Countries like North American nations use rechargeable Smart-Card, offer weekly passes or provide electronic systems for payment within town of metropolis, the user might transfer an app in mobile-phones and make an account that might prompt them to buy a ticket that may be counted upon boarding a bus. Even supposing the general public buses are providing minimalistic satisfactory services, there is a desire for sensible and trustworthy system. The main issue seasoned by the travelers is unexpected waiting times at bus stops, no return of small 
balance, ignorance to provide seat to alternative passengers, etc. Hence, to provide an agile and sleek ticket-collection expertise, they've got planned the smart application that may mechanically allot the seats to traveler, will reserve price ticket online and payment are cashless.Overall promoting digitalization and smart cities initiative.This got us thinking in improving and making our algorithm more efficient andmore frequently we could use the concept of data mining and machine learning. The algorithm we chose to make this efficient is Predictive parsing algorithm. This will learn how to use the app for each individual users according to time and day of the week. This would make the app easily usable and also provide quick service to the user. Predictive Parsing Algorithm is used for predicting the destination of the user depending upon the Source of the user. Thus the research done on these relatable and relevant subjects enables to make this particular project more efficient and more polished in so many ways.

\section{SYSTEM ARCHITECTURE}

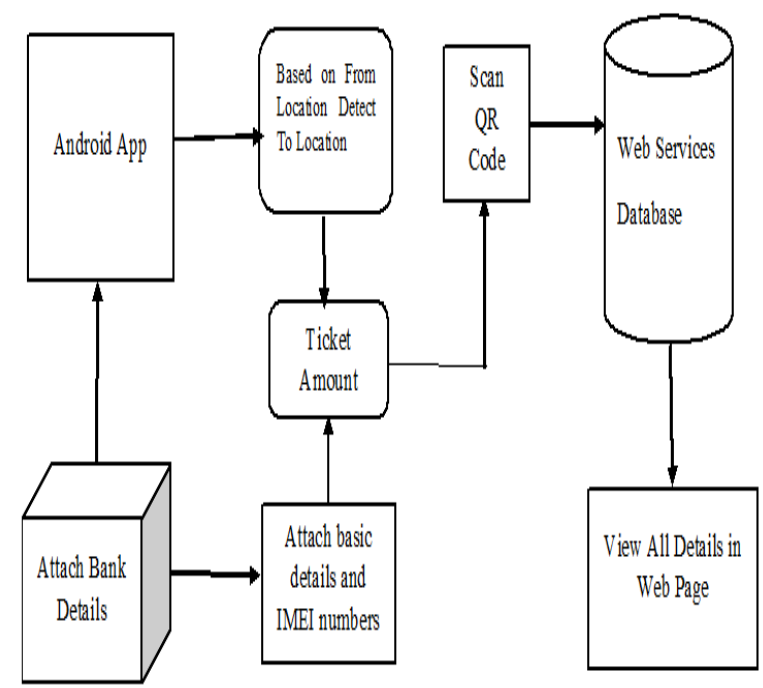

Fig1. System Architecture of the system

\section{A. Login Module}

In login module the details of the passengers are recorded. Username and Password are entered in this module. For new users, Username, Age, Email ID, Mobile Number fields are required to be filled. User must also enter the IMEI numbers of their mobile for tracking issues. This module leads to the next module. Only if verified at this stage a user can go ahead using the facilities.

\section{B. Linking Module}

In this module the bank account is linked with the current user. So once the ticket is confirmed the specified amount will be directly reduced from the Bank account. This module is also responsible for making sure that the user has enough funds in the account to be able to board the bus and pay the ticket charges. Amount remaining in the wallet after the deduction will be displayed to the user.

\section{Area Selection Module}

Depending upon the current location a list of destination stops will be suggested to the user. These locations are known by mining the travelling history of a passenger. This is done through predictive parsing algorithm of Data mining. This makes the selection process easier. The area selection module is also linked with the linking module. This is because only after an area is selected the fee validation is done by the system

\section{Ticket Generation}

After the area selection depending upon the seat availability of the busses, the ticket will be confirmed for the user. A message of confirmation would be sent to the registered mobile number of the user. The ticket is generated for a specific user to a specific bus only. Users boarding the wrong bus will be alerted and will have to board at the next earliest stop and will have to do the booking process again. This makes sure that the seat count on the server is maintained. At the time of boarding the bus the user has to scan the QR code present in the bus through the app which then directly deducts the amount from the bank.

\section{E. Checking Module}

In this module the ticket defaulters are identified. The IMEI numbers of all the users are present in the database. Each bus is equipped with aGPS and an IMEI checker. Now the app will enable GPS services of the user's mobile phone. The GPS is cross verified with the IMEI number of the mobile present inside the bus. Once the passenger enters the bus the IMEI checker inside the bus checks for the IMEI numbers of the mobiles present inside the bus. If the IMEI number of any user inside the bus is not present in the database then that passenger is a ticket defaulter. If the ticket defaulters have enough money in their accounts to pay the fine then it will be deducted then and there. Else their details are in the fine database and they will receive a postdated fine.

\section{METHODOLOGY}

The current system is almost completely handled by physical man power. From collecting currency for the fare, to counting and extracting the total and also accounting for the tickets sold, is done by hand. Now this first hand let experience the amount of errors and tediousness of the current system. A mistake by even one member of the hierarchy results in string of errors. This leads to a lot of the employees losing out from their very own pocket.In current system RFID Reader is employed to browse and tag, however stop point ought to be inputted by traveler in keypad, so quantity are going to debit mechanically from the tag. Once the stop-point is reached, bus stops mechanically and acknowledges the notification. Such arrangement consumes longer just in the case of inputting of tag by each individual. Therefore to beat conventional implementation of ticketing system with accessibility is developed with the additional application to transfer data regarding accident prevalence.

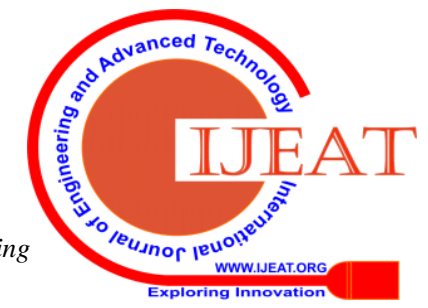


In the proposed system all tickets are paper-less which makes the trail of all those tickets and finances paper-less. A set of 100 tickets per route and price point is called a block. Now the blocks have to be accounted for. And the number of blocks allocated are also limited. So in this system these accounts are easily registered by accessing the database for the booked records. This app along with the web based system helps to reduce the paper trail. This reduces all the human error and makes every single attribute of the whole travel system traceable on a matter of seconds. In this systemQR readers are introduced. Here, an Android application is created for selecting travelling route and generating the ticket amount. After generating amount, the user has to read that QR image. Then automatically app transfers the ticket amount from bank account to the app wallet.Each bus has one QR reader, after reading that the values are automatically stored in the database. Then the user gets the travelling ticket throughan SMS. This system also has a unique feature which is not being used in the current system. By entering the source location the destination is automatically suggested to the user. This is done by predictive parsing algorithm of data mining. Having accessed the user travel history according to the day of the week, a proper suggestion of destination would be popped up. This makes the selection process easier for the user. Ticket defaulter can be easily identified in this system.

The IMEI numbers of all the users are present in the database. Once the passenger enters the bus the IMEI checker inside the bus checks for the IMEI numbers of the mobiles present inside the bus. If the IMEI number of any user inside the bus is not present in the database then that passenger is a ticket defaulter. This system can be established using web application and Android Application. The admin side is handled by web application. MYSQL server stores the details of the user. An APK for the android app is created using Eclipse. The server and the mobile communicate through SOAP protocol. The app contains all the data stored in the database.

\section{TIME BASED PREDICTIVE ALGORITHM}

Using constant input from the user over the course of few days to completely learning travel patterns in a month, this time based predictive algorithm makes it convenient for both the user and the admin in destination selection purposes. The automatic destination selection uses two attributes to learn, the user defined destinations at particular intervals of time. The whole idea here is to let the machine learn how the particular user functions. If he has to go to a certain place frequently at a particular time the algorithm will keep that in check. Hence if eventually the user has to go to a particular place but the timing of his ticket booking is different, then this algorithm will suggest places apart from his frequent travel. For example if a man has to travel to the parliament every day at 9 am the parser understands that he does not have to go to the parliament if he's booking the ticket at $1 \mathrm{pm}$ on the same day. Thus using time and day analysis the destination prediction comes into play. It also learns from his frequent GPS coordinates. On one hand the existing destination predictors have no time to day ratio of comparison. We use this strategy to not only implement the technique but also make it efficient in the highest possible way.

The Algorithm is as follows.

Step 1: Start

Step 2: Define Class Entity withDayCount of Integer type and destination of String type

Step 3: Define Function Input

Step 4: Define Object for ClassEntity and Read the value of ClassEntitydestination

Step 5: Define Function Count with day and time as Parameters

Step 6: Define Object for ClassEntity.

Step 7: Repeat Steps until(Day==day) And (Time == time)

7.1: DayCount $\leftarrow$ DayCount +1

7.2: If DayCount $<7$

Go to Step 3

Step 8: Define Function DestinationPrediction

Step 9: If(count> 7)

Destination $\leftarrow$ destination

Else

Go to Step 3

Step 10: Stop

\section{VII.ADVANTAGES}

- It is completely digital

- It has no trail of physical paper

- It is easy and convenient to use

- It is pre-informative about the status of the required busses

- It provides tracking abilities

- It doesn't require and hardware debugging

- It reduces the requirement of man power

- It creates a trail of travel for the user

- It learns the users day to day travel preferences

- Helps the environment by saving large amount of paper and ink.

- It is secure and exclusive to the user

- It provides flexibility to the user

- It is more convenient than traditional ticketing methods

- The tickets in this app are impossible to lose. That means there is never the excuse of saying that a passenger has lost his or her tickets

- The tickets can be used only by the person for whose identities the tickets have been purchased and reserved for

- There is no charge for losing a ticket

- There is no charge for cancelling a ticket

- The tickets are also engaged and processed in an exceedingly additional timely method, saving labor hours and lowering on person frustration.

- For troubled travelers this may be particularly 
convenient, because it provides period updates on their travel arrangements while not speeding them down.

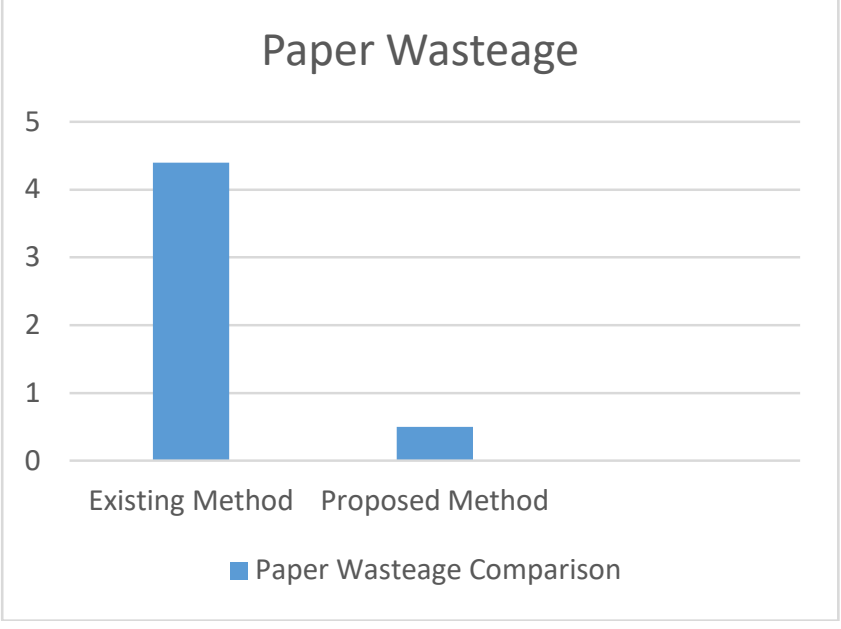

\section{Bus Frequency At Peak Time}

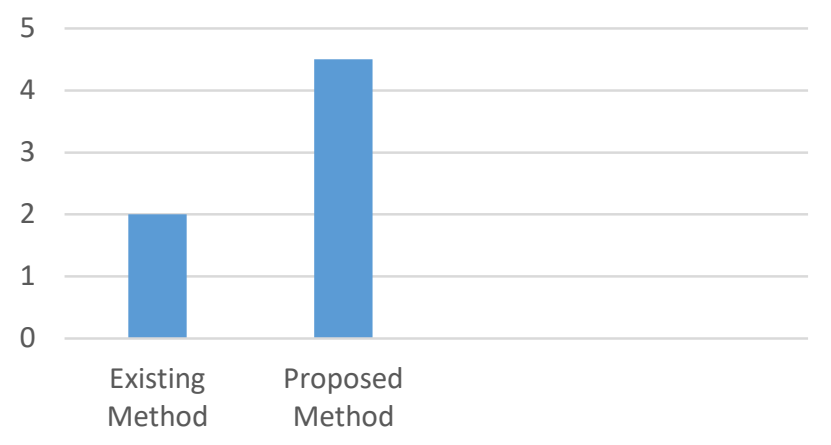

\section{CONCLUSION}

Citizens those is in need of basic services, will have an easy way of booking and commuting in local busses. This automatic system helps in reducing the requirement of man power and also results in almost little to no human errors. This system predicts the destination of the user depending upon the source location, day and time of the week. The exact fare of the ticket will be debited from the user wallet/account avoiding the change issue present in the current system. This system also catches all the ticket defaulters automatically. The number of busses required to run at a specific time can be found depending upon the need of it. Overall, the aim of this systemis to give a smooth and agile ticketing experience to the commuters. This will provide a new ticketing expertise to the passengers' in addition to the currency-less economy. With the new ageing culture of smart-phones and digital wallets this maybe the proper time to move into it so that individuals can get acquainted and improvise the general functionsgiven to rider.

\section{FUTURE ENHANCEMENT}

Infuture, theaboveconceptcanbeutilizedfordigitalized ticket booking system for local busses. This system would result in conductor less and easy way of ticket booking in future. This system will be able to frame a whole new type of organization of ticketing in the metropolitan scale of travel. It will be able to provide a smooth hassle-free and convenient experience to both the consumer and the ticketing authorities.

\section{REFERENCES}

1. S. Kazi, M. Bagasrawala, F. Shaikh And A. Sayyed, "Smart ETicketing System For Public Transport Bus," 2018international Conference On Smart City And Emerging Technology (Icscet), Mumbai, 2018

2. A. Shingare, A. Pendole, N. Chaudhari, P. Deshpande And S.Sonavane, "Gps Supported City Bus Tracking \& Smart Ticketing System," 2015 International Conference On Green Computing And Internet Of Things (Icgciot), Noida, 2015

3. N. Lathia, J. Froehlich And L. Capra, "Mining Public Transport Usage For Personalised Intelligent Transport Systems," 2010 Ieee International Conference On Data Mining, Sydney, Nsw, 2010 S. Sankarananrayanan And P. Hamilton, "Mobile Enabled Bus Tracking And Ticketing System," 2014 2nd International Conference On Information And Communication Technology (Icoict), Bandung, 2014

4. D. K. Sharma And S. R. Ahuja, "A First- Come-First-Serve Bus-Allocation Scheme Using Ticket Assignments," In The Bell System Technical Journal

5. G. Indrajithand K.Vijayakumar, "Automatic Mathematical and Chronological Prediction in Smartphone Keyboard" International Journal of Engineering and Computer Science ISSN: 23197242Volume 5 Issue 5 May 2016, Page No. 16714-16718.

6. K. Vijayakumar and C. Arun, "A Survey on Assessment of Risks in Cloud Migration", International Journal of Applied Engineering Research, ISSN 0973-4562 Vol. 10 No.66 May 2015.

7. K. Vijayakumar and C. Arun, "Continuous Security Assessment of Applications in Cloud Environment", International Journal of Control Theory and Applications, ISSN: 0974-5645 volume No. 9(36), Sep 2016, Page No. 533-541.

\section{AUTHOR PROFILE}

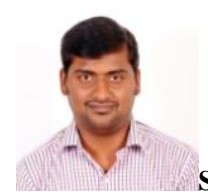

S.Jayakumar- Assistant Professor in the Department of Computer Science And Engineering at SRM Institute of Science And Technology.He has published 6 Papers in National and International conference in the field of Computer Science And Engineering.

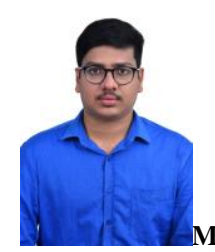

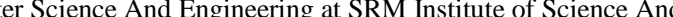
Technology. He has published 4 papers in National and International Conference in the Field of Computer Science And Engineering. 
S.Samundar Ahmed - Student in the Department of Computer Science And Engineering at SRM Institute of Science And Technology. He has published 4 papers in National and International Conference in the Field of Computer Science And Engineering.

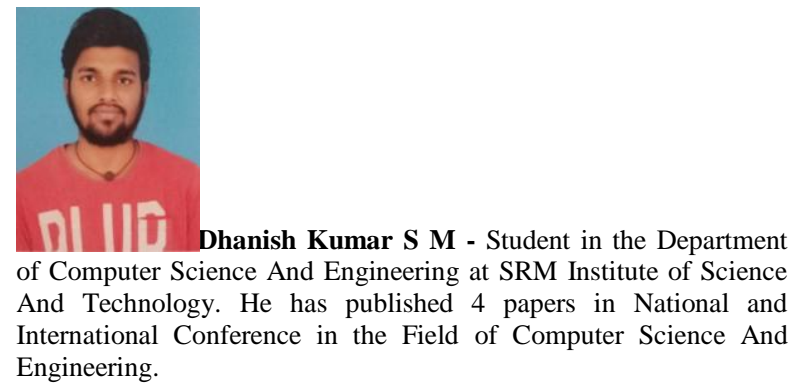
Engineering.

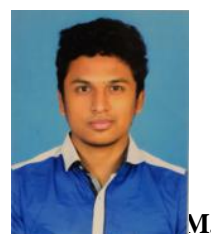
Computer Science And Engineering at SRM Institute of Science And Technology. He has published 4 papers in National and International Conference in the Field of Computer Science And Engineering. 